\title{
On unbounded eigenvalues in particle-transport theory
}

\author{
By C. V. M. van der Mee and C. E. Siewert*, Dept. of Physics and Astronomy,
} The Free University, Amsterdam, The Netherlands

\section{Introduction}

It is sometimes assumed in the field of particle-transport theory that there can be unbounded eigenvalues only for $c=1$, that for $c>1$ there is always an imaginary eigenvalue, that there can be no finite repeated eigenvalues and that there can be no eigenvalues off the real and imaginary axes; in this work we produce explicit counter examples to all of these ideas.

We consider the particle-transport equation $[1,2]$ written as

$$
\mu \frac{\partial}{\partial \tau} \psi(\tau, \mu)+\psi(\tau, \mu)=\frac{c}{2} \sum_{l=0}^{L}(2 l+1) f_{l} P_{l}(\mu) \int_{-1}^{1} P_{l}\left(\mu^{\prime}\right) \psi\left(\tau, \mu^{\prime}\right) \mathrm{d} \mu^{\prime}
$$

where the constants $f_{l}$ are the coefficients in a Legendre representation of the redistribution function

$$
p(\xi)=\sum_{l=0}^{L}(2 l+1) f_{l} P_{l}(\xi), \quad f_{0}=1
$$

We note that $p(\xi) \geqq 0$ for all $\xi \in[-1,1]$ and that $p(\xi)$ is normalized so that

$$
\int_{-1}^{1} p(\xi) \mathrm{d} \xi=2 \text {. }
$$

For steady-state applications in radiative transfer [1] the constant $c$ is the single-scattering albedo and, as such, is confined to the segment $[0,1]$ of the real axis. For applications in the field of neutron-transport theory [2] the constant $c$ represents the mean number of secondary neutrons per collision, and thus any real value of $c \geqq 0$ can be considered. Recalling other applications [3], we note that an equation of the form of Eq. (1) with, in general, complex values of $c$ is obtained after Laplace transformation of the timedependent particle-transport equation.

In this study concerning $c \in[0, \infty)$ we focus our attention on the case $c>1$. Considering now that $c$ is the mean number of neutrons per collision, we note that the redistribution function $p(\xi)$ can be expressed in terms of the individual laws for anisotropic scattering and anisotropic fission. Here we assume only that $p(\xi) \geqq 0$ for all $\xi \in[-1,1]$.

Seeking solutions of Eq. (1) of the form

$$
\psi_{\zeta}(\tau, \mu)=\mathrm{e}^{-\tau / \zeta} \varphi(\zeta, \mu)
$$

\footnotetext{
* Permanent address: Mathematics Department, North Carolina State University, Raleigh, NC 27650 USA.
} 
we find that the "discrete eigenvalues" $\zeta$ must be zeros of the dispersion function

$$
A(z)=1+z \int_{-1}^{1} \psi(\mu) \frac{\mathrm{d} \mu}{\mu-z}, \quad z \notin[-1,1],
$$

where the "characteristic function" $\psi(\mu)$ is given by

$$
\psi(\mu)=\frac{c}{2} \sum_{l=0}^{L}(2 l+1) f_{l} P_{l}(\mu) g_{l}(\mu) .
$$

Here the polynomials $g_{l}(\mu)$ are those used by Chandrasekhar [1], i.e.,

$$
h_{l} \mu g_{l}(\mu)=(l+1) g_{l+1}(\mu)+\lg _{l-1}(\mu)
$$

with $g_{0}(\mu)=1$ and

$$
h_{l}=(2 l+1)\left(1-c f_{l}\right) \text {. }
$$

In this paper we report the findings of our study concerning the number and the location in the complex plane of the zeros of the dispersion function $A(z)$ for the class of problems defined by $c>1, L=2$ and $A(\infty)=0$. For this case we also compute the polynomial solutions of Eq. (1). By focusing our attention on this specific class of problems we demonstrate clear examples of situations not previously encountered for the more studied cases of $c \leqq 1$. For example, we find that $A(z)$ can have a zero of order four at infinity, we find that $\Lambda(z)$ can have a finite double zero and we find that $\Lambda(z)$ can have zeros off the real and imaginary axes.

As we intend to investigate in detail cases that yield unbounded eigenvalues, we summarize here previously reported [4] expressions for the first three terms in an expansion of $\Lambda(z)$ for $|z| \rightarrow \infty$. Thus for $|z| \rightarrow \infty$ we write

$$
\Lambda(z) \rightarrow \Lambda(\infty)+\frac{a_{2}}{z^{2}}+\frac{a_{4}}{z^{4}}+\ldots
$$

where

$$
\begin{aligned}
& A(\infty)=\prod_{l=0}^{L}\left(1-c f_{l}\right), \\
& a_{2}=-c \sum_{l=0}^{L} f_{l} B_{l}
\end{aligned}
$$

and

$$
a_{4}=-c \sum_{i=0}^{L} f_{l} C_{1} .
$$

Here $W_{0}=1, B_{0}=1 / 3, C_{0}=1 / 5$ and, for $l \geqq 0$,

$$
\begin{aligned}
& (2 l+1) W_{l+1}=h_{l} W_{l}, \\
& (2 l+1) B_{l+1}=h_{l} B_{l}+\frac{(l+2)^{2}}{(2 l+5)(2 l+3)} h_{l} W_{l}-\frac{l^{2}}{2 l-1} W_{l-1}
\end{aligned}
$$

and

$$
(2 l+1) C_{l+1}=h_{l} C_{l}+\frac{(l+2)^{2}}{(2 l+5)(2 l+3)} h_{l} T_{l}-\frac{l^{2}}{2 l-1} T_{l-1}
$$

where

$$
T_{l}=B_{l}+\frac{1}{2 l+5}\left[\frac{(l+3)^{2}}{2 l+7}+\frac{(l+2)^{2}}{2 l+3}\right] W_{l}
$$




\section{Unbounded eigenvalues for $L=2$ and $c>1$}

In order to be able to exhibit our analysis of unbounded eigenvalues and the corresponding solutions of Eq. (1) in a particularly explicit way, we restrict our attention here to the case of quadratically anisotropic scattering and fission. We thus consider

$$
p(\xi)=1+3 f_{1} P_{1}(\xi)+5 f_{2} P_{2}(\xi),
$$

and in order to have $p(\xi) \geqq 0$ for $\xi \in[-1,1]$ we consider only those real values of $f_{1}$ and $f_{2}$ that satisfy the necessary and sufficient conditions

$$
\left|f_{1}\right| \leqq \frac{1}{3}\left(1+5 f_{2}\right), \quad-\frac{1}{5} \leqq f_{2} \leqq \frac{1}{10},
$$

and

$$
f_{1}^{2} \leqq \frac{5}{3} f_{2}\left(2-5 f_{2}\right), \quad \frac{1}{10} \leqq f_{2} \leqq \frac{2}{5},
$$

reported by Dawn and Chen [5]. As we consider $c>1$ there clearly are only two ways that $A(z)$ can vanish as $|z| \rightarrow \infty$, viz. $c f_{1}=1$ and $c f_{2}=1$. We therefore proceed to investigate these two possibilities.

A: The case $c f_{1}=1$. We note first of all that Eqs. (18) can be satisfied only for $c \geqq \sqrt{3}$ and $f_{2}$ restricted by

$$
\frac{1}{5}\left[1-\left(1-\frac{3}{c^{2}}\right)^{1 / 2}\right] \leqq f_{2} \leqq \frac{1}{5}\left[1+\left(1-\frac{3}{c^{2}}\right)^{1 / 2}\right], \quad \sqrt{3} \leqq c \leqq 2,
$$

and

$$
\frac{3-c}{5 c} \leqq f_{2} \leqq \frac{1}{5}\left[1+\left(1-\frac{3}{c^{2}}\right)^{1 / 2}\right], \quad 2 \leqq c<\infty .
$$

Considering $c \geqq \sqrt{3}$ and only those values of $f_{2}$ that are allowed by Eqs. (19), we can use the argument principle [6] to deduce that $\Lambda(z)$ has exactly one \pm pair of zeros in addition to the pair that exists at infinity. If we now compute $a_{2}$ from the equations given in the Introduction we find

$$
a_{2}=\frac{c}{3}\left[f_{2}-\frac{9-4 c}{5 c}\right] \text {. }
$$

Thus in the event that

$$
f_{2}=\frac{9-4 c}{5 c} \text {, }
$$

which can happen only for $7 / 4 \leqq c \leqq 2$, it is apparent that both pairs of discrete eigenvalues come together at infinity. Of course if $a_{2} \neq 0$ the sign of $a_{2}$ determines if the finite pair of eigenvalues is real $\left(a_{2}<0\right)$ or imaginary $\left(a_{2}>0\right)$. To summarize our conclusions for the case $c f_{1}=1$ and $f_{2}$ restricted by Eqs. (19), we find that a) $\Lambda(z)$ has a pair of zeros at infinity plus one \pm pair of real zeros for $\sqrt{3} \leqq c<7 / 4, b) \Lambda(z)$ has a pair of zeros at infinity plus one \pm pair of imaginary zeros for $2<\mathrm{c}<\infty$ and c) $\Lambda(z)$ has, for $7 / 4 \leqq c \leqq 2$, one pair of zeros at infinity plus one \pm real pair if $5 c f_{2}<9-4 c$ or one \pm imaginary pair if $5 c f_{2}>9-4 c$; for $5 c f_{2}=9-4 c$ the second pair of zeros becomes unbounded so that for this case $\Lambda(z)$ has all four zeros at infinity. To conclude this case we find the solutions to Eq. (1) corresponding to unbounded eigenvalues to be

$$
\psi_{1}(\tau, \mu)=P_{1}(\mu)
$$


and

$$
\psi_{2}(\tau, \mu)=\tau P_{1}(\mu)+\frac{1}{3(c-1)}\left[P_{0}(\mu)-\frac{2(c-1)}{1-c f_{2}} P_{2}(\mu)\right]
$$

plus, if $5 c f_{2}=9-4 c$,

$$
\psi_{3}(\tau, \mu)=\tau^{2} P_{1}(\mu)+\frac{2 \tau}{3(c-1)}\left[P_{0}(\mu)-\frac{5}{2} P_{2}(\mu)\right]+\frac{1}{c-1} P_{3}(\mu)
$$

and

$$
\begin{aligned}
\psi_{4}(\tau, \mu)= & \tau^{3} P_{1}(\mu)+\frac{\tau^{2}}{c-1}\left[P_{0}(\mu)-\frac{5}{2} P_{2}(\mu)\right]+\frac{3 \tau}{c-1} P_{3}(\mu) \\
& -\frac{12}{7(c-1)}\left[\frac{15}{16(c-1)} P_{2}(\mu)+P_{4}(\mu)\right]
\end{aligned}
$$

B: The case $c f_{2}=1$. For this case we find that Eqs. (18) yield $c \geqq 5 / 2$ and the restrictions

$$
\left|f_{1}\right| \leqq\left[\frac{5}{3 c}\left(2-\frac{5}{c}\right)\right]^{1 / 2}, \quad \frac{5}{2} \leqq c \leqq 10
$$

and

$$
\left|f_{1}\right| \leqq \frac{1}{3}\left(1+\frac{5}{c}\right), \quad 10 \leqq c<\infty .
$$

Again we can use the argument principle [6] to show that $\Lambda(z)$ has two \pm pairs of zeros for all $c \geqq 5 / 2$ provided $f_{1}$ satisfies the conditions

$$
\begin{aligned}
& -\left[\frac{5}{3 c}\left(2-\frac{5}{c}\right)\right]^{1 / 2} \leqq f_{1} \leqq\left[\frac{5}{3 c}\left(2-\frac{5}{c}\right)\right]^{1 / 2}, \quad \frac{5}{2} \leqq c \leqq c_{0}, \\
& -\left[\frac{5}{3 c}\left(2-\frac{5}{c}\right)\right]^{1 / 2} \leqq f_{1} \leqq \frac{13 c-10}{9 c(c-1)}, \quad c_{0} \leqq c \leqq 10,
\end{aligned}
$$

and

$$
-\frac{1}{3}\left(1+\frac{5}{c}\right) \leqq f_{1} \leqq \frac{13 c-10}{9 c(c-1)}, \quad 10 \leqq c \leqq \infty,
$$

where $c_{0} \simeq 3.2603$ is the real solution of

$$
270 c^{3}-1384 c^{2}+1880 c-775=0 .
$$

It is not difficult to show that of these two pairs of zeros, one pair is at infinity and the other is purely imaginary. Considering now the other values of $c \geqq 5 / 2$ and $f_{1}$ allowed by Eqs. (24), but excluded from Eqs. (25), we find that $\Lambda(z)$ has three \pm pairs of zeros for

$$
\frac{13 c-10}{9 c(c-1)}<f_{1} \leqq\left[\frac{5}{3 c}\left(2-\frac{5}{c}\right)\right]^{1 / 2}, \quad c_{0} \leqq c \leqq 10,
$$

and

$$
\frac{13 c-10}{9 c(c-1)}<f_{1} \leqq \frac{1}{3}\left(1+\frac{5}{c}\right), \quad 10 \leqq c<\infty .
$$

Of these three pairs of zeros, we find that one pair is always at infinity. For $27 c f_{1}<55$ we find, in addition to the pair at infinity, that there are one real pair and one purely 
imaginary pair of zeros. For $27 c f_{1}=55$ we find that there are two pairs of zeros at infinity plus one purely imaginary pair of zeros. For $27 c f_{1}>55$ we find, in addition to the pair at infinity, that there can be either two pairs of purely imaginary zeros or two pairs of zeros that are neither real nor imaginary. We have found here, for example, that $A(z)$ has zeros off the real and imaginary axes for $c=9$ and $f_{1}=0.4$. We note that the conclusions drawn on the location of the zeros of $A(z)$ for $27 c f_{1}>55$ were based on computational evidence obtained from the exact solutions of $A(z)=0$ given in Ref. 4 . We note that Davison [7], Kuščer [8] and Protopopescu and Sjöstrand [9] have also found cases where the eigenvalues can be off the real and imaginary axes.

For $c \leqq 1$ the finite zeros of $A(z)$ are known to be simple [10]. For $c>1$ this need not be the case. As the zeros of $A(z)$ occur in \pm and conjugate pairs, as the zeros of $A(z)$ depend, for the considered case of $c f_{2}=1$, continuously on $c$ and $f_{1}$ and as we have computational evidence of the existence of zeros off the real and imaginary axes, we conclude that $A(z)$ can have a pair of double, finite imaginary zeros for $27 c f_{1}>55$ and for suitable values of $c$ and $f_{1}$.

For the case $c f_{2}=1$ we find the appropriate solutions to Eq. (1) to be

$$
\psi_{1}(\tau, \mu)=P_{2}(\mu)
$$

and

$$
\psi_{2}(\tau, \mu)=\tau P_{2}(\mu)+\frac{3}{5}\left[-\frac{2}{3\left(1-c f_{1}\right)} P_{1}(\mu)-P_{3}(\mu)\right]
$$

plus, if $27 c f_{1}=55$,

and

$$
\psi_{3}(\tau, \mu)=\tau^{2} P_{2}(\mu)+\frac{6}{5} \tau\left[\frac{9}{14} P_{1}(\mu)-P_{3}(\mu)\right]+\frac{24}{35}\left[\frac{3}{8(c-1)} P_{0}(\mu)+P_{4}(\mu)\right]
$$

$$
\begin{aligned}
\psi_{4}(\tau, \mu)= & \tau^{3} P_{2}(\mu)+\frac{9}{5} \tau^{2}\left[\frac{9}{14} P_{1}(\mu)-P_{3}(\mu)\right]+\frac{72}{35} \tau\left[\frac{3}{8(c-1)} P_{0}(\mu)+P_{4}(\mu)\right] \\
& +\frac{8}{7}\left[\frac{729}{1120(c-1)} P_{1}(\mu)-\frac{4}{5} P_{3}(\mu)-P_{5}(\mu)\right]
\end{aligned}
$$

\section{Acknowledgement}

One of the authors (CES) wishes to express his gratitude to J. W. Hovenier and the Free University of Amsterdam for their kind hospitality during recent visits to The Netherlands. This work was supported in part by the U.S. National Science Foundation.

\section{References}

[1] S. Chandrasekhar, Radiative transfer, Oxford University Press, London (1950).

[2] K. M. Case and P. F. Zweifel, Linear transport theory, Addison-Wesley, Reading, Mass. (1967).

[3] R. L. Bowden and C. D. Williams, J. Math. Phys. 5, 1527 (1964).

[4] C. E. Siewert, J. Math. Phys. 21, 2468 (1980).

[5] T. Dawn and I. Chen, Nucl. Sci. Eng. 72, 237 (1979).

[6] L. V. Ahlfors. Complex analysis. McGraw-Hill, New York (1953).

[7] B. Davison, Milne problem in a multiplying medium with a linearly anisotropic scattering, Chalk River Report CRT 358, Ontario (1946).

[8] 1. Kuščer, Nucl. Sci. Eng., 38, 175 (1969). 
[9] V. Protopopescu and N. G. Sjöstrand, On the solution of the dispersion equation for monoenergetic neutron transport with quadratically anisotropic scattering, Chalmers University of Technology Report CTH-RF-36, Göteborg (1981).

[10] J. K. Shultis and T. R. Hill, Nucl. Sci. Eng. 59, 53 (1976).

\begin{abstract}
The dispersion function derived from particle-transport theory is analyzed for the specific case of a three-term redistribution law in order to define those $c>1$ cases for which there can be either one or two pairs of unbounded eigenvalues, and the elementary solutions corresponding to the unbounded eigenvalues are reported.
\end{abstract}

\title{
Zusammenfassung
}

Die Dispersions-Funktion, die von der Teilchen-Transport-Theorie erhalten wurde, wird analysiert für den besonderen Fall eines dreigliedrigen Neuverteilungsgesetzes, um die Fälle $c>1 \mathrm{zu}$ definieren, für die entweder ein oder zwei Paare von unbegrenzten Eigenwerten existieren. Die zugehörigen elementaren Lösungen werden angegeben.

(Received: November 12, 1982) 